\title{
Ultrasonic based structural damage detection using combined finite element and model Lamb wave propagation parameters in composite materials
}

\author{
B. S. Ben • S. H. Yang $\cdot$ Ch. Ratnam $\cdot$ B. A. Ben
}

Received: 24 November 2011 / Accepted: 30 October 2012/Published online: 16 November 2012

(C) The Author(s) 2012. This article is published with open access at Springerlink.com

\begin{abstract}
This paper presents a combined finite element and model Lamb waves propagation parameters method as a tool for structural health monitoring in composite materials. Modal analysis allows identifying the mode conversions induced by the defects. A simulation combining a lossless finite element approach and Lamb wave propagation parameter for finding natural frequencies and mode shapes of the structures in undamaged and damaged condition is proposed. This analysis is performed on two carbon-fiber-reinforced plastic bars in both undamaged and damaged state, where the two damaged states are (1) having a cut partway through the bar, perpendicular to the long axis of the bar and (2) having a circular hole. The lamb wave propagation parameters are calibrated using the ultrasonic pulse generator test setup. The natural frequencies for the theoretical, finite element and experimental results are compared and close agreement is found between the frequencies obtained experimentally and computationally.
\end{abstract}

\footnotetext{
B. S. Ben $(\bowtie)$

Institute of mechanical engineering technology,

Kyungpook National University,

Daegu, South Korea

e-mail: satishben@gmail.com

B. S. Ben

e-mail: satishben@yahoo.com

S. H. Yang

School of Mechanical Engineering,

Kyungpook National University,

Daegu, South Korea

C. Ratnam • B. A. Ben

Department of Mechanical Engineering (AUCE),

Andhra University,

Visakhapatnam, India
}

Keywords Lamb wave · Propagation parameters · Damage detection $\cdot$ Structural health monitoring

\section{Introduction}

Identification of change in natural frequencies and mode shapes of a freely vibrating damaged plate with respect to its undamaged state has traditionally been a popular method for damage identification. This method can be very much useful when a combined finite element and acoustic emission (AE) method is used.

Acoustic emission has been extensively used during the past 30 years for nondestructive evaluation of damage as well as for the study of fracture behavior of materials. AE monitoring is considered to be a very attractive NDT method as it provides real-time information for damage evolution and propagation within the material. Most studies so far have used AE descriptors such as counts, amplitude, and energy to characterize the development of damage mechanisms. Furthermore, additional efforts have been directed towards the use of multivariable data analysis utilizing statistical pattern recognition algorithms [1-4], with interesting results. Although descriptor-based $\mathrm{AE}$ techniques are relatively easy to perform, they ignore $\mathrm{AE}$ waveforms, which also carry useful information. Thus, another group of studies were dedicated to the more difficult task of waveform processing of AE signals.

The vast majority of the above studies were performed in the time domain. Some researchers though insisted that valuable information was lying in the frequency domain as well [5-7]. The most important outcome of the research in frequency domain was the suggestion that each failure mechanism is distinguished by a different peak frequency obtained by fast Fourier transform analysis of the acquired waveforms. 
Many authors have also investigated the interaction of Lamb modes with a single defect like crack, notch, or circular cavity. Some of them used analytical [8] or semianalytical [9] resolutions, whereas others chose a finite element or a boundary element modeling [10-13]. Analytical or semi-analytical resolutions can be used when the geometry of the defect is regular and when the problem presents symmetries. Finite or boundary element modeling allows studying the interaction of Lamb modes with irregular defects but require respecting spatial and temporal discretization which can cause numerical problems at high frequency-thickness product. Indeed, the number of freedom degrees of this problem becomes rapidly huge and cannot be solved only by using a finite element model.

Ben et al. [14] has presented a simulation combining a planar, lossless finite element analysis with Lamb wave propagation parameter for isotropic materials. In the present work, a similar kind of method has been proposed for finding natural frequencies and mode shapes of structures in undamaged and damaged condition for composite materials. The natural frequencies obtained from the proposed method are compared theoretically and with ANSYS model and close agreement is found between the frequencies obtained experimentally and computationally.

\section{Experimental setup and test specimens}

The schematic diagram of the experimental setup is shown in Fig. 1. The test specimen is clamped at one end on cantilever support fixed on basement and the transducers are placed on the test specimen at a distance of $160 \mathrm{~mm}$ from each other. A coupling fluid is used between transducers and the test specimen for getting good results. The pitch-catch RF test method, which uses a dual-element, point-contact, ultrasonic transducer in which one element transmits a burst of acoustic waves into the test part, and a separate element receives the sound propagated across the test piece between the transducer tips, as shown in Fig. 2. Both the actuation and the data acquisition are performed using a portable Panametrics-NDT ${ }^{\mathrm{TM}}$ EPOCH 4PLUS and a desktop PC running Scanview plus as a virtual controller.

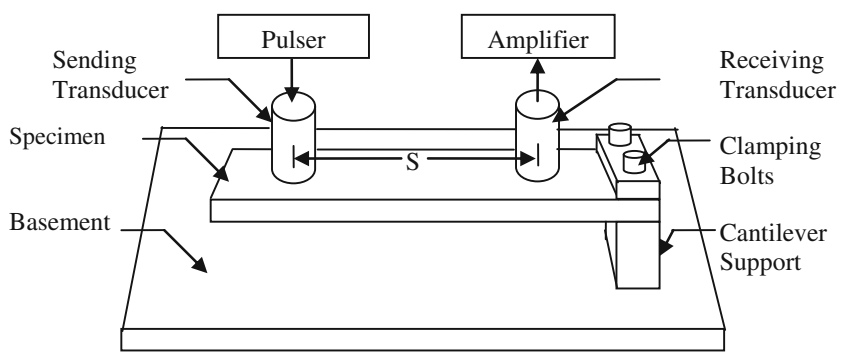

Fig. 1 Schematic representation of experimental setup

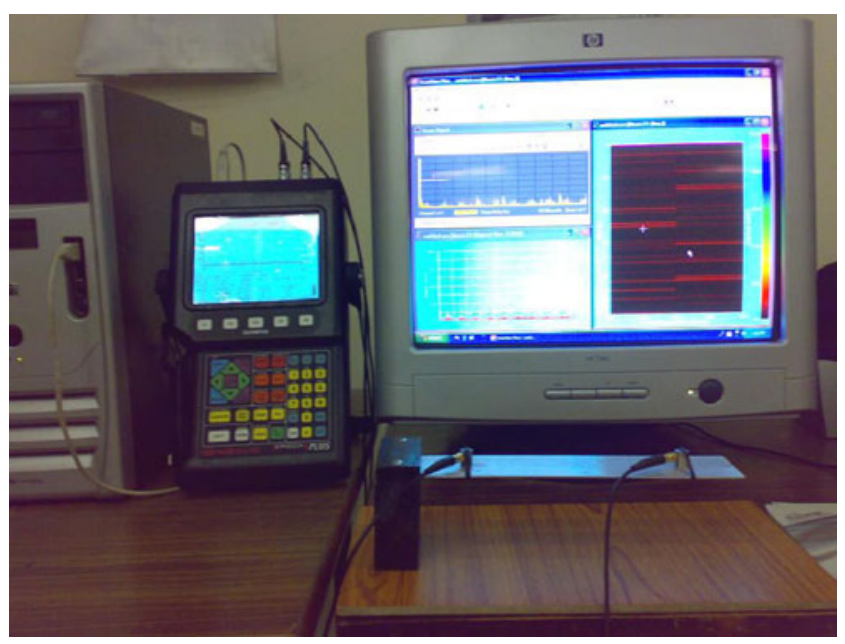

Fig. 2 Experimental setup

The specimen considered are two carbon-fiber-reinforced plastic (CFRP) plates (plates A and B), with the dimensions $250 \times 50 \times 2 \mathrm{~mm}$ in undamaged state and the same plates, plate $\mathrm{A}$, cut $(30 \times 2 \times 2 \mathrm{~mm})$ at a distance $120 \mathrm{~mm}$ away from free end and other plate $\mathrm{B}$, with a $8 \mathrm{~mm}$ diameter through hole at a distance $110 \mathrm{~mm}$ away from free end in damaged state shown in Fig. 3.

\section{Calibration of ultrasonic pulse generator for optimal lamb wave generation}

The Panametrics-NDT ${ }^{\mathrm{TM}}$ EPOCH 4PLUS is capable of producing ultrasonic sound waves and is equipped with four channels, i.e., Device, Pulser, Receiver and Waveform. Each channel is having editable parameters tabulated below.

\begin{tabular}{llllll} 
Channels & \multicolumn{2}{l}{ Editable parameters } & & & \\
Device & Unit & Angle & Thickness & & \\
Pulser & Mode & Energy & Damping & Wave type & Frequency \\
Receiver & Gain & Broad band & Low pass & High pass & By pass \\
Waveform & Range & Rectification & Offset & & \\
\end{tabular}

The optimal driving frequency for specimens is obtained by varying different editable parameters shown in the above table and Fig. 4. shows the calibration of ultrasonic pulse generator for CFRP specimen based on fitted peak value and similarly it is calibrated for various material. Based on the calibration, various curves are plotted between pulser frequency and signal amplitude at receiver for different materials to find optimal driving frequency and it is shown in Fig. 5. A histogram representation of percentage amplitude of waveform at constant gain which is used to attain a trend line for optimal driving frequency for different materials is shown in Fig. 6 and the percent amplitude of the waveform 


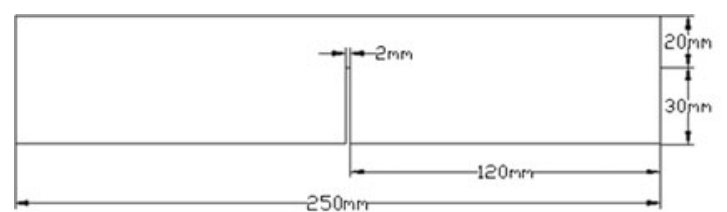

a

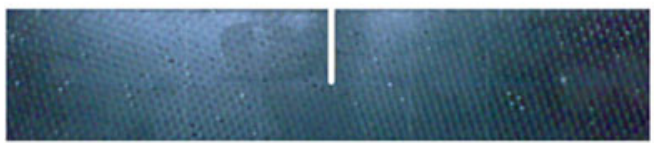

C

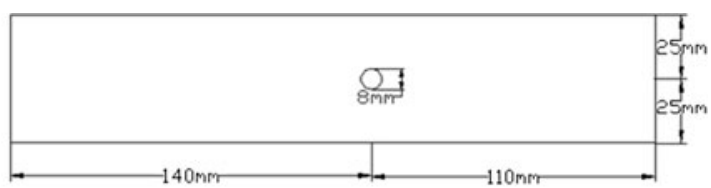

b

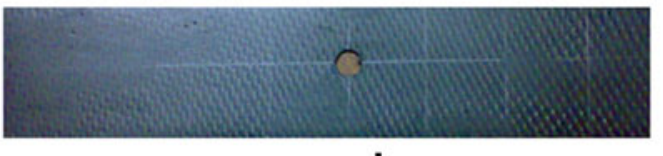

d

Fig. 3 a Schematics diagram of damaged plate having a cut; $\mathbf{b}$ schematics diagram of damaged plate having a hole; $\mathbf{c}$ test specimen having a cut; $\mathbf{d}$ test specimen having a hole

at various pulser frequencies are taken as bin range $(0-$ $820 \mathrm{kHz}$ ) at constant gain $55 \mathrm{~dB}$ for different materials.

The frequency of the transducer to be used is proportional to the acoustic impedance of the layer. Materials such as graphite or fiberglass with low impedance require lower frequency transducers than metal skin layers. The frequencies in the range of 100-460 kHz have been useful in most of the testing and it observed that the higher frequencies can be used for thinner and metallic layers.

Increasing the driving voltage proportionately increases the magnitude of the Lamb wave strain. From Fig. 6, the percentage amplitude of the waveform in the range of 55-70 has been useful in the most of the testing. Driving the actuating probe at 5-10 V produced a 10-25 $\mathrm{mV}$ response due to the Lamb wave at the sensing probe, with $1-5 \mathrm{mV}$ of static noise. Increasing the amplitude also increases the signal to noise ratio to yield a clearer signal. Higher voltage however also tended to increase the drift in the signal, which deteriorated the resolution capabilities of the data acquisition system.

The signal shapes that are tested, pure sinusoidal shapes appear to excite Lamb wave harmonics the most efficiently, since they are periodic, smooth, and have comparatively quick rise times to their peak amplitude as compared to a parabolic shape. A Hanning window helps to narrow the bandwidth further to focus the maximum amount of energy into the desired actuating frequency with the least "spillover" from neighboring frequencies.

To first order, the wave velocity increases with the square root of the modulus, i.e., an increase in modulus slightly speeds the wave. An increase in the density would have the opposite effect however by slowing wave velocity. The effect of the Poisson's ratio is probably the most complicated, as it appears in the most terms, however to first order, small changes seem to have little to no effect on the wave velocity. The most straightforward parameter is the thickness of the specimen, which has a linear relationship with the Lamb wave velocity. The thicker the specimen the quicker the speed and the higher the dispersion rate for a given driving frequency.

\section{Methodology}

In the present work, identification of change in natural frequencies and mode shapes of a freely vibrating damaged plate with respect to its undamaged state has been used for damage identification.

The vibration is a characteristic property of the structure and it depends on the distribution of mass and stiffness in the structure. The basic material property Young's modulus which is of interest in many manufacturing and research applications can be determined quickly and easily through computations based on Lamb wave velocities [15-18]. Young's modulus is determined using the relations given below

$$
\begin{aligned}
& E_{1}=\frac{V_{L}^{2} \rho(1+v)(1-2 v)}{1-v} \\
& E_{2}=\frac{V_{T}^{2} \rho(1+v)(1-2 v)}{1-v}
\end{aligned}
$$

where $V_{L}$ and $V_{T}$ are longitudinal and transverse velocity of Lamb waves traveling in the material respectively, $\rho$ is material density and $v$ is Poisson's ratio.

The velocities of undamaged and damaged plates are determined experimentally using ultrasonic pulse generator test setup and they are tabulated in Table 1. The Young's modulus is calculated by using Eq. (1) and thus obtained Young's modulus is substituted in the [D] matrix of the finite element model for finding natural frequencies and mode shapes of cantilever plate. ANSYS model has been used for validation purpose and the discussion is carried out in results section.

\section{Finite element model for free vibration analysis of a composite plate}

The oscillatory motion is a characteristic property of the structure and it depends on the distribution of mass 

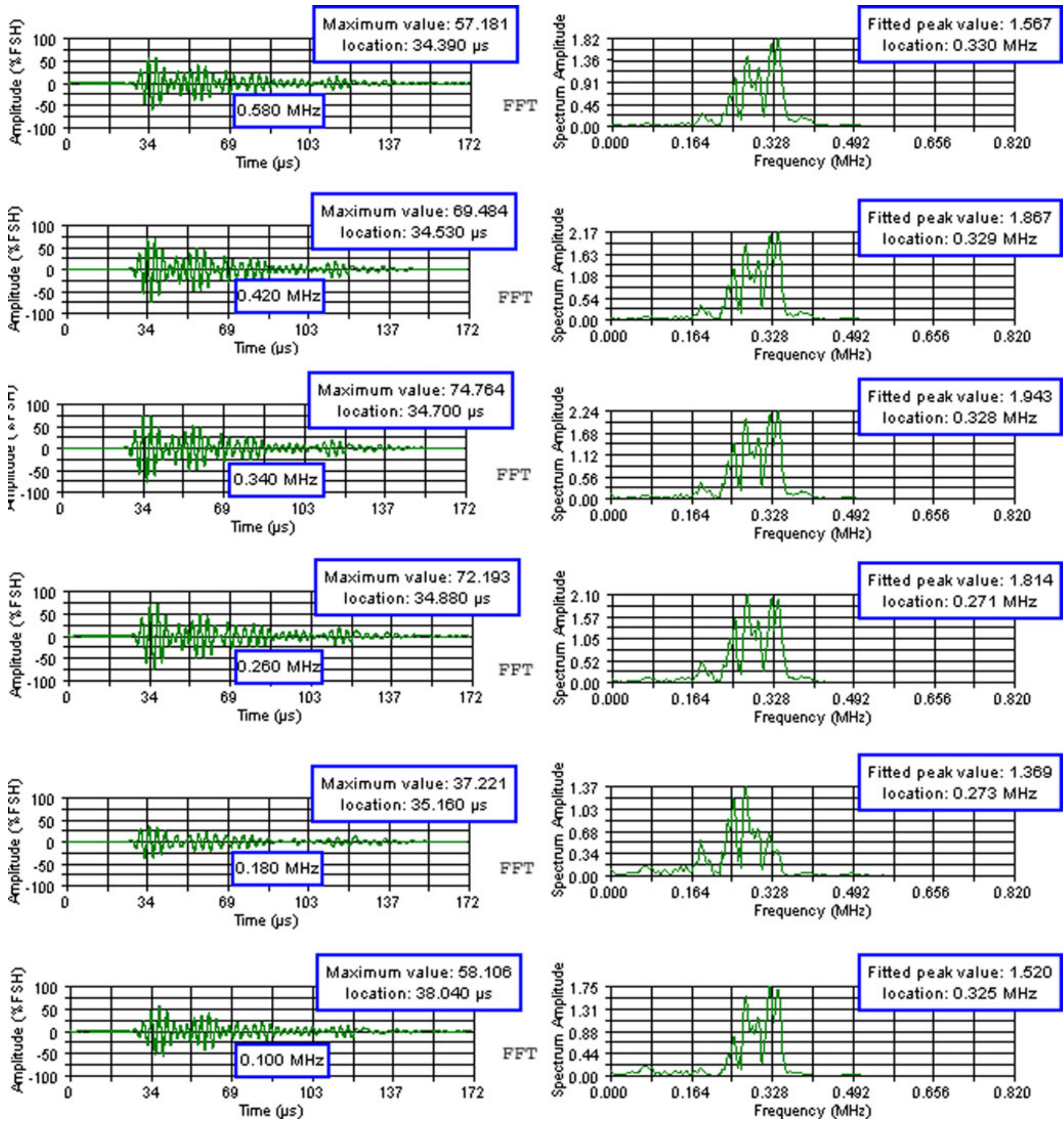

Fig. 4 Calibration of ultrasonic pulse generator for CFRP specimen at various Pulsar frequencies

and stiffness in the structure. If damping is present, the amplitude of oscillations will decay progressively and if the magnitude of damping exceeds a certain critical value, the oscillatory character of the motion will cease altogether. On the other hand, if damping is absent, the oscillatory motion will continue indefinitely, with the amplitudes of oscillations depending on the initially imposed disturbance or displacement. The oscillatory motion occurs at certain frequencies known as natural frequencies or characteristic values, and it follows welldefined deformation pattern known as mode shapes or characteristic modes. The study of such free vibration is very important in finding the dynamic response of elastic structures. 


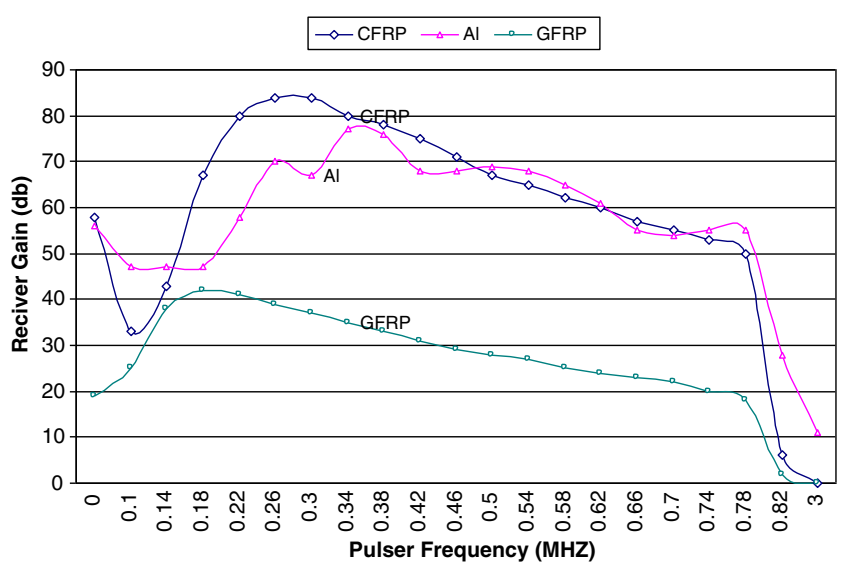

Fig. 5 Optimal driving frequency selection for different materials

By assuming the external force vector $\vec{p}$ to be zero and the displacement to be harmonic as:

$\vec{Q}=\vec{Q} \cdot e^{i \omega t}$

and the free vibration equation is given by:

$$
\left[[\mathbf{k}]-\omega^{2}[\mathbf{M}]\right] \underline{\vec{Q}}=\vec{O}
$$

where $\vec{Q}$ represents the amplitude of the displacement $\vec{Q}$ (mode shape or eigen vector) and $\omega$ denotes the natural frequency of vibration. Equation (2) is called a linear algebraic eigenvalue problem since neither $[\mathbf{k}]$ nor $[\mathbf{M}]$ is a function of the circular frequency $\omega$, and it will have a nonzero solution for $\vec{Q}$ provided that the determinant of coefficient matrix $\left([\mathbf{k}]-\omega^{2}[\mathbf{M}]\right)$ is zero, i.e.,

$\left|[\mathbf{k}]-\omega^{2}[\mathbf{M}]\right|=0$

where $[\mathbf{k}]$ is stiffness matrix and $[\mathbf{M}]$ is mass matrix. As said above, the oscillatory motion is a characteristic property of

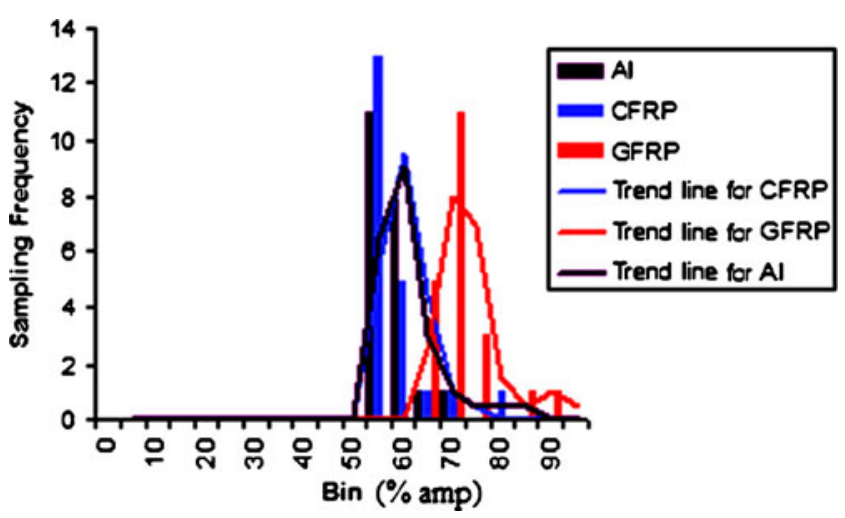

Fig. 6 Histogram representation of percent amplitude of waveform at constant gain the structure and it depends on the distribution of mass and stiffness in the structure, so the stiffness and mass matrix are derived as follows.

The plate bending developed in this section is shown in Fig. 7 where $x, y$, and $z$ describe the global coordinate of the plate; $u, v$, and $w$ are the displacements, and $h$ is the plate thickness. The $x y$ plane is parallel to the midsurface plane prior to deflection.

The displacement of any point in the plate can be expressed as

$u=u(x, y, z)$

$v=v(x, y, z)$

$w=w(x, y, z)$

That is the in plane displacement $u$ and $v$ vary through the plate thickness as well as within the $x y$ plane while the transverse displacement $w$ remains constant through the plate thickness $[19,20]$. In order to interpolate the displacement using shape functions and nodal displacements, two different interpolations are needed: one interpolation within the $x y$ plane and the other in the $z$-axis. For the $x y$ plane interpolation, shape function $N_{i}(x, y)$ are used where subscript I varies depending on the number of nodes on the $x y$ plane. On the other hand, shape function $H_{j}(z)$ are used for interpolation along the $z$-axis, where subscript $j$ varies depending on the number of nodes along the plate thickness. Because two inplane displacement are functions of $x, y$, and $z$, both shape functions are used while the transverse displacement uses shape functions $N_{i}(x, y)$ using isoparametric element with mapping of $\xi, \eta$-plane onto $x y$-plane and $\zeta$-axis to $z$-axis, the three displacements can be expressed as

$u=\sum_{i=1}^{N_{1}} \sum_{j=1}^{N_{2}} N_{i}(\xi, \eta) H_{j}(\zeta) u_{i j}$

$v=\sum_{i=1}^{N_{1}} \sum_{j=1}^{N_{2}} N_{i}(\xi, \eta) H_{j}(\zeta) v_{i j}$

$w=\sum_{i=1}^{N_{1}} N_{i}(\xi, \eta) w_{i}$

In which $N_{1}$ and $N_{2}$ are the number of nodes in $x y$-plane $(\xi, \eta$ plane) and $z$-axis ( $\zeta$-axis), respectively. In additional, the first subscript for $u$ and $v$ denotes the node numbering in terms of $x y$ plane ( $\xi, \eta$ plane) and the second subscript indicates the node numbering in terms of $z$-axis ( $\zeta$-axis). In the present study, $N_{1}=$ 4 and $N_{2}=2$ that is four-node quadrilateral shape function are employed for the $x y$ plane ( $\xi, \eta$ plane) interpolation and linear 
Table 1 Experimentally calibrated velocities and Young's modulus

\begin{tabular}{|c|c|c|c|c|c|c|c|c|}
\hline \multirow[t]{2}{*}{ Specimen } & \multicolumn{4}{|c|}{ Undamaged condition } & \multicolumn{4}{|c|}{ Damaged condition } \\
\hline & $V_{L}(\mathrm{~m} / \mathrm{s})$ & $V_{T}(\mathrm{~m} / \mathrm{s})$ & $E_{1}(\mathrm{Gpa})$ & $E_{2}(\mathrm{Gpa})$ & $V_{L}(\mathrm{~m} / \mathrm{s})$ & $V_{T}(\mathrm{~m} / \mathrm{s})$ & $E_{1}(\mathrm{Gpa})$ & $E_{2}(\mathrm{Gpa})$ \\
\hline Plate A & 9,321 & 2,347 & 70.11 & 4.44 & 9,078 & 2,218 & 66.5 & 3.97 \\
\hline Plate B & 9,286 & 2,185 & 69.58 & 3.85 & 9,050 & 2,041 & 66.09 & 3.36 \\
\hline
\end{tabular}

shape function are employed for the $z$-axis ( $\zeta$-axis) interpolation. Nodal displacement $u_{i 1}$ and $v_{i 1}$ are displacement on the bottom surface of the plate element and $u_{i 2}$ and $v_{i 2}$ are displacement on the top surface. As seen in Eqs. (8) through (10), there is no rotational degree of freedom for the present plate bending element.

In the present formulation, both bending strain energy and transverse shear strain energy are included. The bending strains and transverse shear strain are expressed in terms of displacements.

$$
\begin{gathered}
\left\{\varepsilon_{b}\right\}=\left\{\begin{array}{c}
\varepsilon_{x} \\
\varepsilon_{y} \\
\gamma_{x y}
\end{array}\right\}=\left[\begin{array}{ccc}
\frac{\partial}{\partial x} & 0 & 0 \\
0 & \frac{\partial}{\partial y} & 0 \\
\frac{\partial}{\partial y} & \frac{\partial}{\partial x} & 0
\end{array}\right]\left\{\begin{array}{c}
u \\
v \\
w
\end{array}\right\} \\
\left\{\varepsilon_{s}\right\}=\left\{\begin{array}{l}
\gamma_{y z} \\
\gamma_{x z}
\end{array}\right\}=\left[\begin{array}{ccc}
\frac{\partial}{\partial z} & 0 & \frac{\partial}{\partial x} \\
0 & \frac{\partial}{\partial z} & \frac{\partial}{\partial y}
\end{array}\right]\left\{\begin{array}{c}
u \\
v \\
w
\end{array}\right\}
\end{gathered}
$$

Where $\left\{\varepsilon_{b}\right\}$ is the bending strain and $\left\{\varepsilon_{s}\right\}$ is the transverse shear strain. The normal strain along the plate thickness $\varepsilon_{z}$ is omitted here.

Substitution of displacement, Eqs. (8) through (10), into the kinematic equations, Eqs. (11) and (12), with $N_{1}=4$ and $N_{2}=2$ expresses both bending and shear strain in the following way.

$\left\{\varepsilon_{S}\right\}=\left[B_{b}\right]\left\{d^{e}\right\}$

Fig. 7 Plate element with displacement degrees of freedom
Where

$\left[B_{b}\right]=\left[\left[B_{b 1}\right] \quad\left[B_{b 2}\right] \quad\left[B_{b 3}\right] \quad\left[B_{b 4}\right]\right]$

$\left[B_{b}\right]=\left[\begin{array}{ccccc}H_{1} \frac{\partial N_{i}}{\partial x} & 0 & H_{2} \frac{\partial N_{i}}{\partial x} & 0 & 0 \\ 0 & H_{1} \frac{\partial N_{i}}{\partial y} & 0 & H_{2} \frac{\partial N_{i}}{\partial y} & 0 \\ H_{1} \frac{\partial N_{i}}{\partial y} & H_{1} \frac{\partial N_{i}}{\partial x} & H_{2} \frac{\partial N_{i}}{\partial y} & H_{2} \frac{\partial N_{i}}{\partial x} & 0\end{array}\right]$

$\left\{d^{e}\right\}=\left\{\left\{d_{1}^{e}\right\} \quad\left\{d_{2}^{e}\right\} \quad\left\{d_{1}^{e}\right\} \quad\left\{d_{2}^{e}\right\}\right\}^{T}$

$\left\{d_{i}^{e}\right\}=\left\{\begin{array}{lllll}u_{i 1} & v_{i 1} & u_{i 2} & v_{i 2} & w_{i}\end{array}\right\}$

$\left\{\varepsilon_{s}\right\}=\left[B_{s}\right]\left\{d^{e}\right\}$

Where

$\left[B_{s}\right]=\left[\begin{array}{llll}{\left[B_{s 1}\right]} & {\left[B_{s 2}\right]} & {\left[B_{s 3}\right]} & {\left[B_{s 4}\right]}\end{array}\right]$

$\left[B_{s i}\right]=\left[\begin{array}{ccccc}N_{i} \frac{\partial H_{1}}{\partial z} & 0 & N_{i} \frac{\partial H_{2}}{\partial z} & 0 & \frac{\partial N_{i}}{\partial x} \\ 0 & N_{i} \frac{\partial H_{1}}{\partial z} & 0 & N_{i} \frac{\partial H_{2}}{\partial z} & \frac{\partial H_{2}}{\partial y}\end{array}\right]$

The constitutive equation for the isotropic material is

$\left\{\sigma_{b}\right\}=\left[D_{b}\right]\left\{\varepsilon_{b}\right\}$

$\left\{\sigma_{b}\right\}=\left\{\begin{array}{lll}\sigma_{x} & \sigma_{y} & \tau_{x y}\end{array}\right\}^{T}$

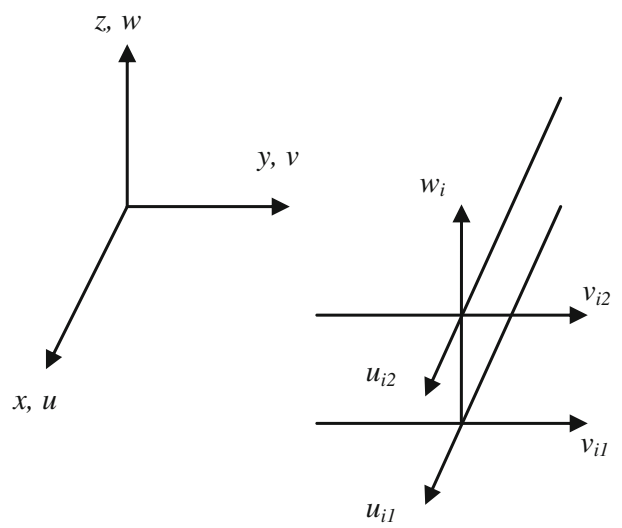

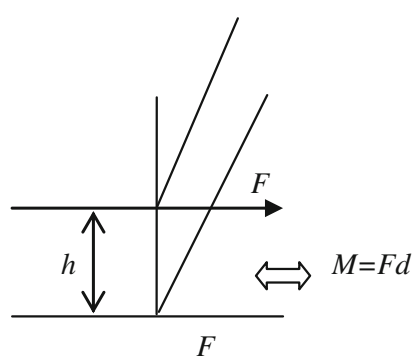


Fig. 8 a Time amplitude signal of undamaged and damaged plate having a cut; $\mathbf{b}$ time amplitude signal of undamaged and damaged plate having a hole

Table 2 Natural frequencies of first four mode shapes of plate A

Fig. 9 a First bending mode at $35.08 \mathrm{~Hz}$, b first torsion at $136.55 \mathrm{~Hz}$, c second bending at $195.90 \mathrm{~Hz}$, d second torsion at $463.44 \mathrm{~Hz}$
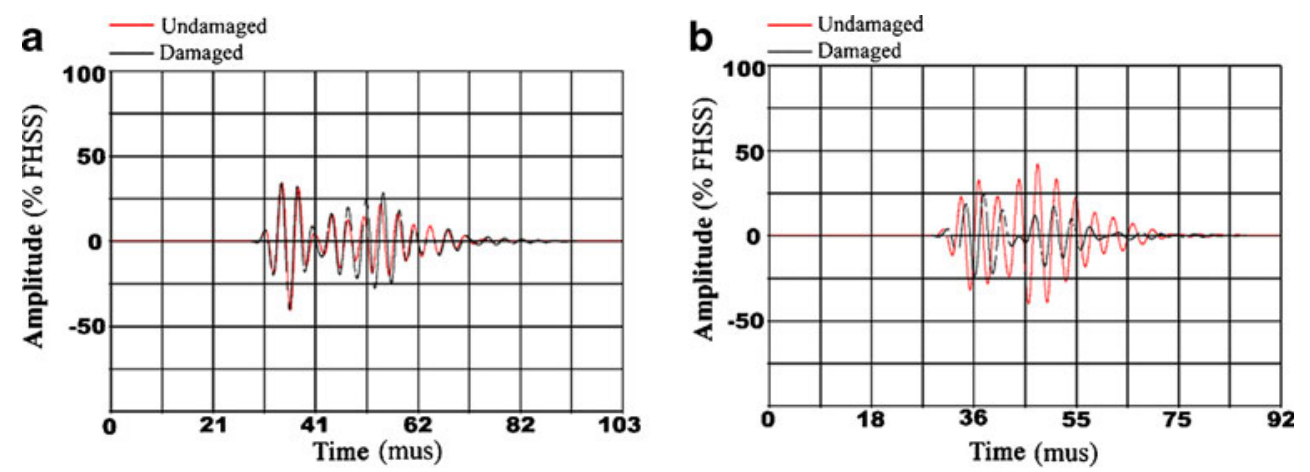

\begin{tabular}{lcccccrrr}
\hline \multirow{2}{*}{ Mode (Hz) } & \multicolumn{2}{l}{ Undamaged condition } & & & \multicolumn{2}{l}{ Damaged condition } \\
& Theoretical & Present work & ANSYS & & Theoretical & Present work & ANSYS \\
\hline First bending & 36.23 & 38.68 & 38.77 & - & 38.66 & 35.08 \\
First bending & 164.14 & 166.24 & 167.86 & - & 135.27 & 136.55 \\
First bending & 240.26 & 242.79 & 243.40 & - & 195.83 & 195.90 \\
First bending & 557.03 & 558.02 & 560.95 & - & 462.20 & 463.44 \\
\hline
\end{tabular}

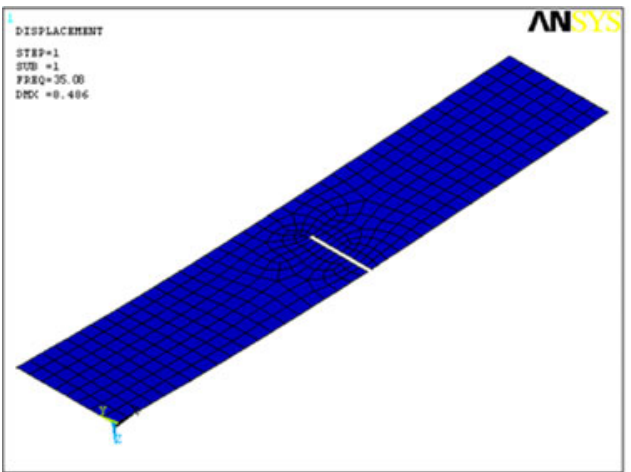

a

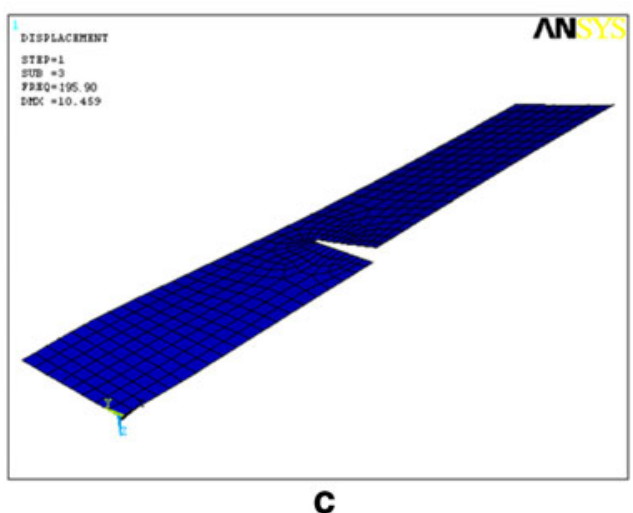

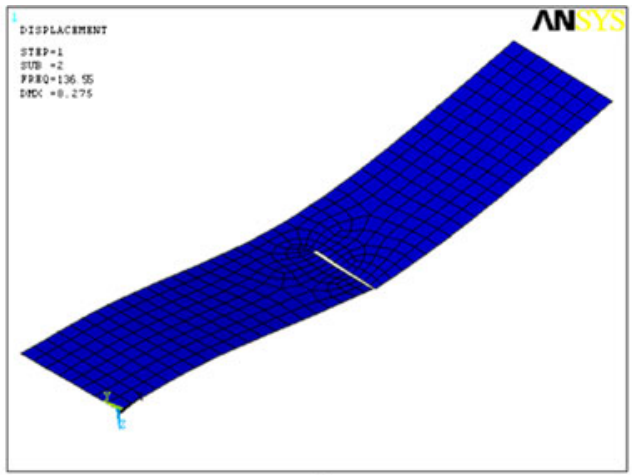

b

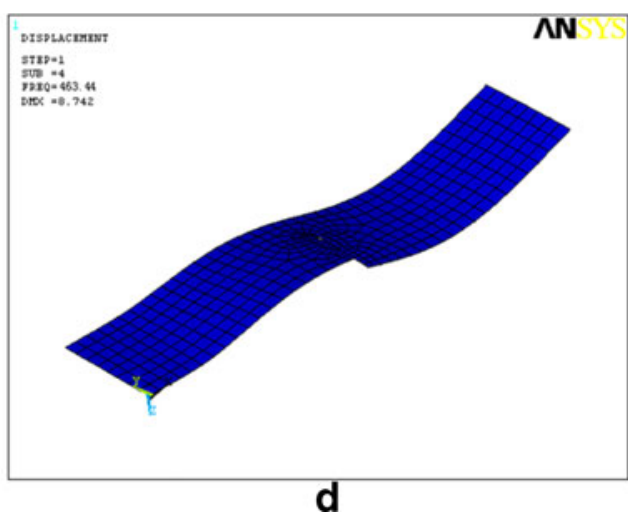


Table 3 Natural frequencies of first four mode shapes of plate B

\begin{tabular}{|c|c|c|c|c|c|c|}
\hline \multirow[t]{2}{*}{ Mode $(\mathrm{Hz})$} & \multicolumn{3}{|c|}{ Undamaged condition } & \multicolumn{3}{|c|}{ Damaged condition } \\
\hline & Theoretical & Present work & ANSYS & Theoretical & Present work & ANSYS \\
\hline First bending & 36.23 & 38.22 & 38.61 & - & 38.25 & 39.00 \\
\hline First bending & 164.14 & 166.89 & 167.38 & - & 144.94 & 145.00 \\
\hline First bending & 240.26 & 241.65 & 242.35 & - & 242.86 & 242.88 \\
\hline First bending & 557.03 & 557.17 & 558.13 & - & 484.94 & 485.67 \\
\hline
\end{tabular}

$\left[D_{b}\right]=\frac{E}{1-v^{2}}\left[\begin{array}{ccc}1 & v & 0 \\ v & 1 & 0 \\ 0 & 0 & \frac{1-v}{2}\end{array}\right]$

For the bending components

$\left\{\sigma_{s}\right\}=\left[D_{s}\right]\left\{\varepsilon_{s}\right\}$

Where

$\left\{\sigma_{s}\right\}=\left\{\begin{array}{ll}\tau_{y z} & \tau_{x z}\end{array}\right\}^{T}$

$\left[D_{s}\right]=\frac{E}{2(1+v)}\left[\begin{array}{ll}1 & 0 \\ 0 & 1\end{array}\right]$

Fig. 10 a First bending mode at $39.00 \mathrm{~Hz}$, b first torsion at $145.00 \mathrm{~Hz}, \mathbf{c}$ second bending mode at $242.88 \mathrm{~Hz}$, d second torsion at $485.67 \mathrm{~Hz}$
Where Eq. (23) is the material property matrix for the plane stress condition as usually assumed for the plate bending theory and for a fibrous composite, the material property matrices is given by

$\left[D_{b}\right]=\left[\begin{array}{ccc}D_{11} & D_{12} & 0 \\ D_{12} & D_{22} & 0 \\ 0 & 0 & D_{33}\end{array}\right]$

In which

$D_{11}=\frac{E_{1}}{1-v_{12} v_{21}}$

(26) $\quad D_{12}=\frac{E_{1} v_{21}}{1-v_{12} v_{21}}$

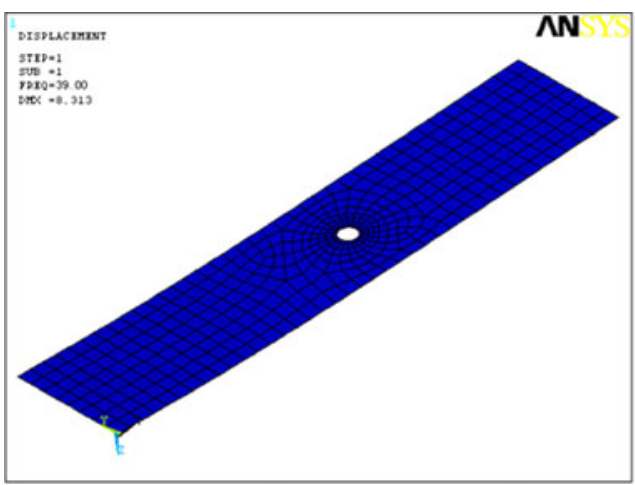

a

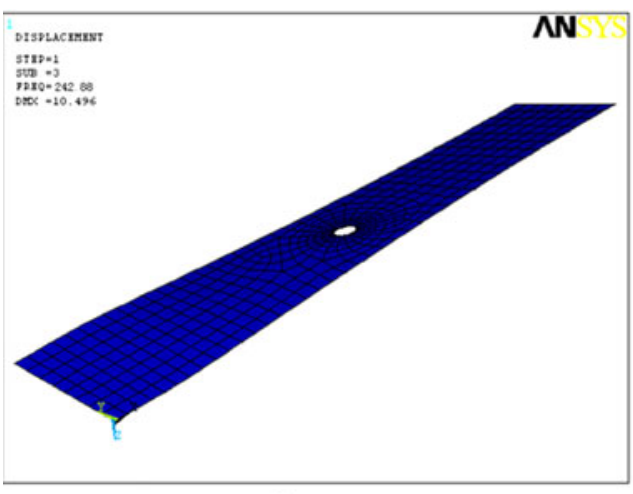

C

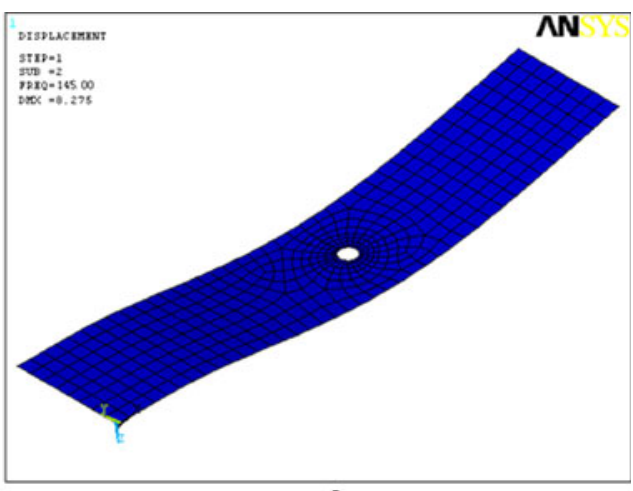

b

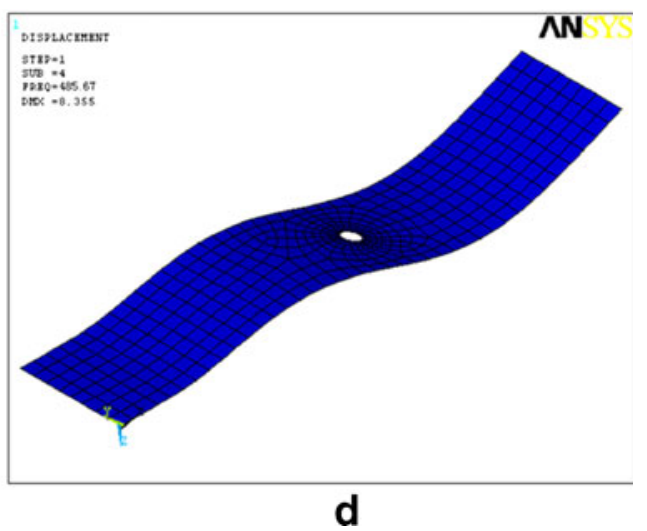


$D_{22}=\frac{E_{2}}{1-v_{12} v_{21}}$

$D_{33}=G_{12}$

And

$\left[D_{s}\right]=\left[\begin{array}{cc}G_{13} & 0 \\ 0 & G_{12}\end{array}\right]$

Here, 1 and 2 denote the longitudinal and transverse directions of the composite, respectively. Further $E$ is the elastic modulus, $G_{i j}$ the shear modulus of the $i-j$ plane and $v_{i j}$ is Poisson's ratio for strain in the $j$ direction when stressed in the $i$ direction. There are five independent material properties for Eqs. (27) through (32) because of the reciprocal relation

$\frac{v_{12}}{E_{1}}=\frac{v_{21}}{E_{2}}$

by minimum potential energy approach the stiffness matrix of the element $\left[\mathbf{k}^{(e)}\right]$ is expressed as:

$\left[k^{(e)}\right]=\int_{\Omega^{e}}\left[B_{b}\right]^{T}\left[D_{b}\right]\left[B_{b}\right] \partial \Omega+\int_{\Omega^{e}}\left[B_{s}\right]^{T}\left[D_{s}\right]\left[B_{s}\right] \partial \Omega$

where $\Omega$ is the plate domain.

Similarly, the mass matrix is given by

$\left[M^{(e)}\right]=\frac{\rho A t}{9}\left[\begin{array}{llll}4 & 2 & 1 & 2 \\ 2 & 4 & 2 & 1 \\ 1 & 2 & 4 & 2 \\ 2 & 1 & 2 & 4\end{array}\right]$

where $A$ element area, $t$ thickness of element and $\rho$ density of material. Using the above discussed method, natural frequencies are computed for composite plates in their undamaged and damaged state. The computation has been carried out using Mathematica ${ }^{\mathrm{TH}}$ software and the inputs for the solution have been taken from the experimental data discussed in the previous sections.

\section{Results and discussion}

Figure 8 shows the time amplitude waveform of plates $\mathrm{A}$ and $\mathrm{B}$ both in damaged and undamaged condition. From Fig. 8, it is observed that the change in phase of wave form occurs between undamaged plates and damaged plates. Therefore, the velocity of the lamb wave also varies for undamaged plates and damaged plates. The velocities of the test specimens are calibrated experimentally and the values are tabulated in Table 1.

The material properties considered in the present work, Young's modulus $\left(E_{1}\right.$ and $\left.E_{2}\right)$ from Table 1 , density $(\rho)$ $1,599.92 \mathrm{~kg} / \mathrm{m}^{3}$, Poisson's ratio $(v)$, and Shear modulus (G) from [21]. In ANSYS model, the Young's modulus of the undamaged plates from Table 1, respectively, is used for both the damaged plate and undamaged plate analysis.

Table 2 shows the natural frequencies of the first four mode shapes for plate A and corresponding mode shapes of the plate in damaged case is shown in Fig. 9. Similarly, Table 3 shows the natural frequencies of the first four mode shapes for plate B and corresponding mode shapes of the plate in damaged case is shown in Fig. 10.

The difference between the natural frequencies of plate $B$ in damaged condition and undamaged condition is very small when compared the same with plate A. Therefore, the stiffness of a structure is less affected by a central hole than a cut on the edge.

\section{Conclusion}

The present work explored combining a lossless finite element approach and Lamb wave propagation parameter for finding natural frequencies and mode shapes of cantilever composite plates in undamaged and damaged condition and they are compared theoretically and with ANSYS model. A very good correlation is found between the proposed method and the other methods used in this work.

The method presented in this work can be used successfully for structural health monitoring for fiber-reinforced composite materials using the change in natural frequencies in undamaged and damaged condition.

Acknowledgments This work was supported by the Priority Research Centers Program through the National Research Foundation of Korea (NRF) funded by the Ministry of Education, Science and Technology (2010-0020089)

Open Access This article is distributed under the terms of the Creative Commons Attribution License which permits any use, distribution, and reproduction in any medium, provided the original author(s) and the source are credited.

\section{References}

1. Kostopoulos V et al (2003) On the identification of the failure mechanisms in oxide/oxide composites using acoustic emission. NDT\&E Int 36(8):571-580

2. Ohtsu M, Ono K (1998) Pattern recognition analysis of acoustic emissionfrom uni-directional carbon-fibre epoxy composites by using autoregressive modeling. J Acoust Emission 6(1):61-71

3. Pappas YZ, Markopoulos YP, Kostopoulos V (1998) Failure mechanisms analysis of 2D carbon/carbon using acoustic emission monitoring. NDT\&E Int 31(3):157-163

4. Mizutani Y, Nagashima K et al (2000) Fracture mechanism characterization of cross-ply carbon fibre composites using acoustic emission analysis. NDT\&E Int 33:101-110

5. Groot PJ, Winjen PA, Janseen BF (1995) Real-time frequency determination of acoustic emission for different fracture 
mechanisms in carbon/epoxy composites. Compos Sci Technol $55: 405-412$

6. Barre S (1994) On the use of acoustic emission to investigate damage mechanisms in glass-fibre-reinforced polypropylene. Compos Sci Technol 52:369-376

7. Vaidya UK, Raju PK (1993) Identification of failure modes of carbon-carbon composites at various processing stages using the acoustic emission technique. NCA-V 16/AMD 172:153-161

8. Lamb GT (2003) Wave scattering from a circular partly throughthickness hole in a plate. Wave Motion 37:63-80

9. Castaings M, Le Clezio E, Hosten B (2002) Modal decomposition method for modeling the interaction of Lamb waves with cracks. J Acoust Soc Am 112(6):2567-2582

10. Diligent O, Lowe MJS, Cawley (2001) Reflection and scattering of the S0 Lamb mode from circular defects in plates. Rev Prog Quant Nondestruct Eval 20:134-141

11. Alleyne DN, Cawley P (1992) The interaction of Lamb waves with defects. IEEE Trans Ultrason Ferroelectr Freq Control 39:381-397

12. Guo N, Cawley P (1993) The interaction of Lamb waves with delaminations in composite laminates. J Acoust Soc Am 94 (4):2240-2246
13. Galan JM, Abascal R (2005) Boundary element solution for the bidimensional scattering of guided waves in laminate plates. Comput Struct 83:740-757

14. Ben BS et al (2009) Structural damage detection using combined finite element and model Lamb wave propagation parameters. J Mech Eng Sci C 223:769-777

15. McIntire P. (ed.) (1991) Nondestructive testing handbook. American Society for Nondestructive Testing 7: 398-402

16. Krautkramer J, Krautkramer H (1983) Ultrasonic Testing of Materials, Berlin, Heidelberg, New York Third Edition: 580-587

17. Lynnworth LC, Papadakis EP, Fowler KA (1977) Ultrasound propagation measurements and applications. Int J Nondestruct Test 5:71-115

18. Lynnworth LC (1969) Acoustical nomograms for the elastic properties of engineering materials. Ultrasonics 254-256

19. Owen DRJ, Li ZH (1987) A refined analysis of laminated plates by finite element displacement method-I fundamental and static analysis. Comput Struct 26:907-914

20. Kwon YW (1989) Finite element analysis of crack closure in plate bending. Comput Struct 32:1439-1445

21. Hyer MW (1998) Stress analysis of fiber-reinforced composite materials. McGraw-Hill, New York, pp 58-61 